\title{
On the partial finite sums of the reciprocals of the Fibonacci numbers
}

\author{
Andrew YZ Wang* and Peibo Wen
}

${ }^{\text {*Correspondence: }}$ yzwang@uestc.edu.cn

School of Mathematical Sciences, University of Electronic Science and Technology of China, Chengdu, 611731, P.R. China

\begin{abstract}
In this article, we obtain two interesting families of partial finite sums of the reciprocals of the Fibonacci numbers, which substantially improve two recent results involving the reciprocal Fibonacci numbers. In addition, we present an alternative and elementary proof of a result of Wu and Wang.
\end{abstract}

MSC: $11 \mathrm{~B} 39$

Keywords: Fibonacci numbers; partial sums; reciprocal

\section{Introduction}

The Fibonacci sequence [1], Sequence A000045 is defined by the linear recurrence relation

$$
F_{n}=F_{n-1}+F_{n-2} \quad \text { for } n \geq 2 \text {, }
$$

where $F_{n}$ is the $n$th Fibonacci number with $F_{0}=0$ and $F_{1}=1$. There exists a simple and non-obvious formula for the Fibonacci numbers,

$$
F_{n}=\frac{1}{\sqrt{5}}\left(\frac{1+\sqrt{5}}{2}\right)^{n}-\frac{1}{\sqrt{5}}\left(\frac{1-\sqrt{5}}{2}\right)^{n} .
$$

The Fibonacci sequence plays an important role in the theory and applications of mathematics, and its various properties have been investigated by many authors; see [2-5].

In recent years, there has been an increasing interest in studying the reciprocal sums of the Fibonacci numbers. For example, Elsner et al. [6-9] investigated the algebraic relations for reciprocal sums of the Fibonacci numbers. In [10], the partial infinite sums of the reciprocal Fibonacci numbers were studied by Ohtsuka and Nakamura. They established the following results, where $\lfloor\cdot\rfloor$ denotes the floor function.

Theorem 1.1 For all $n \geq 2$,

$$
\left\lfloor\left(\sum_{k=n}^{\infty} \frac{1}{F_{k}}\right)^{-1}\right\rfloor= \begin{cases}F_{n-2}, & \text { if } n \text { is even; } \\ F_{n-2}-1, & \text { if } n \text { is odd. }\end{cases}
$$

Theorem 1.2 For each $n \geq 1$,

$$
\left\lfloor\left(\sum_{k=n}^{\infty} \frac{1}{F_{k}^{2}}\right)^{-1}\right\rfloor= \begin{cases}F_{n} F_{n-1}-1, & \text { if } n \text { is even; } \\ F_{n} F_{n-1}, & \text { if } n \text { is odd. }\end{cases}
$$


Further, Wu and Zhang $[11,12]$ generalized these identities to the Fibonacci polynomials and Lucas polynomials and various properties of such polynomials were obtained.

Recently, Holliday and Komatsu [13] considered the generalized Fibonacci numbers which are defined by

$$
G_{n+2}=a G_{n+1}+G_{n}, \quad n \geq 0
$$

with $G_{0}=0$ and $G_{1}=1$, and $a$ is a positive integer. They showed that

$$
\left\lfloor\left(\sum_{k=n}^{\infty} \frac{1}{G_{k}}\right)^{-1}\right\rfloor= \begin{cases}G_{n}-G_{n-1}, & \text { if } n \text { is even and } n \geq 2 \\ G_{n}-G_{n-1}-1, & \text { if } n \text { is odd and } n \geq 1\end{cases}
$$

and

$$
\left\lfloor\left(\sum_{k=n}^{\infty} \frac{1}{G_{k}^{2}}\right)^{-1}\right\rfloor= \begin{cases}a G_{n} G_{n-1}-1, & \text { if } n \text { is even and } n \geq 2 ; \\ a G_{n} G_{n-1}, & \text { if } n \text { is odd and } n \geq 1 .\end{cases}
$$

More recently, Wu and Wang [14] studied the partial finite sum of the reciprocal Fibonacci numbers and deduced that, for all $n \geq 4$,

$$
\left\lfloor\left(\sum_{k=n}^{2 n} \frac{1}{F_{k}}\right)^{-1}\right\rfloor=F_{n-2} .
$$

Inspired by Wu and Wang's work, we obtain two families of partial finite sums of the reciprocal Fibonacci numbers in this paper, which significantly improve Ohtsuka and Nakamura's results, Theorems 1.1 and 1.2. In addition, we present an alternative proof of (1.5).

\section{Reciprocal sum of the Fibonacci numbers}

We first present several well known results on Fibonacci numbers, which will be used throughout the article. The detailed proofs can be found in [5].

Lemma 2.1 Let $n \geq 1$, we have

$$
F_{n}^{2}-F_{n-1} F_{n+1}=(-1)^{n-1}
$$

and

$$
F_{a} F_{b}+F_{a+1} F_{b+1}=F_{a+b+1}
$$

if $a$ and $b$ are positive integers.

As a consequence of (2.2), we have the following result.

Corollary 2.2 For all $n \geq 1$, we have

$$
F_{2 n}=F_{n-1} F_{n}+F_{n} F_{n+1},
$$




$$
\begin{aligned}
& F_{2 n+1}=F_{n}^{2}+F_{n+1}^{2}, \\
& F_{2 n+1}=F_{n-1} F_{n+1}+F_{n} F_{n+2} .
\end{aligned}
$$

It is easy to derive the following lemma and we leave the proof as a simple exercise.

Lemma 2.3 For each $n \geq 1$, we have

$$
F_{n+1} F_{n+2}-F_{n-1} F_{n}=F_{2 n+1} .
$$

We now establish two inequalities on Fibonacci numbers which will be used later.

Lemma 2.4 If $n \geq 6$, then

$$
F_{n-2} F_{n-1}>F_{n+1} \text {. }
$$

Proof It is easy to see that

$$
\begin{aligned}
F_{n-2} F_{n-1}-F_{n+1} & =F_{n-2} F_{n-1}-\left(F_{n-1}+F_{n}\right) \\
& =F_{n-2} F_{n-1}-F_{n-1}-\left(F_{n-2}+F_{n-1}\right) \\
& =\left(F_{n-2}-2\right) F_{n-1}-F_{n-2} .
\end{aligned}
$$

Since $n \geq 6, F_{n-2}-2>1$. So

$$
F_{n-2} F_{n-1}-F_{n+1}>F_{n-1}-F_{n-2}>0,
$$

which completes the proof.

Lemma 2.5 For each $n \geq 3$, we have

$$
F_{3 n-1}\left(F_{n}+F_{n-3}\right)>F_{n-2} F_{n-1} F_{n} F_{n+1} .
$$

Proof Applying (2.2), we get

$$
F_{3 n-1}=F_{n-1} F_{2 n-1}+F_{n} F_{2 n} .
$$

Thus

$$
F_{3 n-1}\left(F_{n}+F_{n-3}\right) \geq\left(F_{n-1} F_{2 n-1}+F_{n} F_{2 n}\right) F_{n}>F_{n}^{2} F_{2 n}>F_{n-1} F_{n} F_{2 n} .
$$

Employing (2.3), we have

$$
F_{2 n}>F_{n} F_{n+1}>F_{n-2} F_{n+1} \text {, }
$$

which yields the desired equation (2.8). 
The following are some inequalities on the sum of reciprocal Fibonacci numbers.

Proposition 2.6 For all $n \geq 2$, we have

$$
\sum_{k=n}^{2 n} \frac{1}{F_{k}}>\frac{1}{F_{n-2}+1}
$$

Proof For all $k \geq 2$,

$$
\begin{aligned}
\frac{1}{F_{k-2}+1}-\frac{1}{F_{k}}-\frac{1}{F_{k-1}+1} & =\frac{F_{k}-F_{k-2}-1}{F_{k}\left(F_{k-2}+1\right)}-\frac{1}{F_{k-1}+1} \\
& =\frac{\left(F_{k-1}-1\right)\left(F_{k-1}+1\right)-F_{k} F_{k-2}-F_{k}}{\left(F_{k-2}+1\right)\left(F_{k-1}+1\right) F_{k}} \\
& =\frac{F_{k-1}^{2}-F_{k} F_{k-2}-1-F_{k}}{\left(F_{k-2}+1\right)\left(F_{k-1}+1\right) F_{k}} .
\end{aligned}
$$

Invoking (2.1), we obtain $F_{k-1}^{2}-F_{k} F_{k-2}=(-1)^{k}$. Therefore,

$$
\frac{1}{F_{k-2}+1}-\frac{1}{F_{k}}-\frac{1}{F_{k-1}+1}=\frac{(-1)^{k}-1-F_{k}}{\left(F_{k-2}+1\right)\left(F_{k-1}+1\right) F_{k}}
$$

Now we have

$$
\begin{aligned}
\sum_{k=n}^{2 n} \frac{1}{F_{k}} & =\frac{1}{F_{n-2}+1}-\frac{1}{F_{2 n-1}+1}+\sum_{k=n}^{2 n} \frac{(-1)^{k-1}+1+F_{k}}{\left(F_{k-2}+1\right)\left(F_{k-1}+1\right) F_{k}} \\
& >\frac{1}{F_{n-2}+1}-\frac{1}{F_{2 n-1}+1}+\sum_{k=n}^{2 n} \frac{1}{\left(F_{k-2}+1\right)\left(F_{k-1}+1\right)} \\
& >\frac{1}{F_{n-2}+1}+\frac{1}{\left(F_{n-2}+1\right)\left(F_{n-1}+1\right)}-\frac{1}{F_{2 n-1}+1}
\end{aligned}
$$

Because of (2.5), we have

$$
\begin{aligned}
F_{2 n-1}+1-\left(F_{n-2}+1\right)\left(F_{n-1}+1\right) & =F_{2 n-1}-F_{n-2} F_{n-1}-F_{n-2}-F_{n-1} \\
& =F_{n-2}^{2}+F_{n-1} F_{n+1}-F_{n} \\
& >0 .
\end{aligned}
$$

Thus, we arrive at

$$
\sum_{k=n}^{2 n} \frac{1}{F_{k}}>\frac{1}{F_{n-2}+1}
$$

This completes the proof.

Proposition 2.7 Assume that $m \geq 2$. Then, for all even integers $n \geq 4$, we have

$$
\sum_{k=n}^{m n} \frac{1}{F_{k}}<\frac{1}{F_{n-2}}
$$


Proof By elementary manipulations and (2.1), we deduce that

$$
\frac{1}{F_{k-2}}-\frac{1}{F_{k}}-\frac{1}{F_{k-1}}=\frac{(-1)^{k}}{F_{k-2} F_{k-1} F_{k}}, \quad k \geq 3 .
$$

Hence, for $n \geq 3$, we have

$$
\sum_{k=n}^{m n} \frac{1}{F_{k}}=\frac{1}{F_{n-2}}-\frac{1}{F_{m n-1}}+\sum_{k=n}^{m n} \frac{(-1)^{k-1}}{F_{k-2} F_{k-1} F_{k}} .
$$

Since $n$ is even,

$$
\sum_{k=n}^{m n} \frac{(-1)^{k-1}}{F_{k-2} F_{k-1} F_{k}}<0
$$

from which we conclude that

$$
\sum_{k=n}^{m n} \frac{1}{F_{k}}<\frac{1}{F_{n-2}} .
$$

The proof is complete.

Proposition 2.8 If $n \geq 5$ is odd, then

$$
\sum_{k=n}^{2 n} \frac{1}{F_{k}}<\frac{1}{F_{n-2}} .
$$

Proof It is straightforward to check that the statement is true when $n=5$.

Now we assume that $n \geq 7$. Since $n$ is odd, we have

$$
\sum_{k=n+1}^{2 n} \frac{(-1)^{k-1}}{F_{k-2} F_{k-1} F_{k}}<0 .
$$

Applying (2.7) and (2.6) yields

$$
\begin{aligned}
\frac{1}{F_{n-2} F_{n-1} F_{n}}-\frac{1}{F_{2 n-1}} & <\frac{1}{F_{n} F_{n+1}}-\frac{1}{F_{2 n-1}} \\
& =\frac{F_{2 n-1}-F_{n} F_{n+1}}{F_{n} F_{n+1} F_{2 n-1}} \\
& =-\frac{F_{n-2} F_{n-1}}{F_{n} F_{n+1} F_{2 n-1}} \\
& <0 .
\end{aligned}
$$

Employing (2.11) and the above two inequalities, (2.12) follows immediately.

Proposition 2.9 Let $m \geq 3$ be given. If $n \geq 3$ is odd, we have

$$
\sum_{k=n}^{m n} \frac{1}{F_{k}}>\frac{1}{F_{n-2}} .
$$


Proof It is easy to see that

$$
\sum_{k=3}^{3 m} \frac{1}{F_{k}}>\frac{1}{F_{1}}
$$

thus (2.13) holds for $n=3$. Now we assume that $n \geq 5$.

Based on (2.11) and using the fact $n$ is odd, we have

$$
\sum_{k=n}^{m n} \frac{1}{F_{k}}=\frac{1}{F_{n-2}}+\frac{1}{F_{n-2} F_{n-1} F_{n}}-\frac{1}{F_{n-1} F_{n} F_{n+1}}-\frac{1}{F_{m n-1}}+\sum_{k=n+2}^{m n} \frac{(-1)^{k-1}}{F_{k-2} F_{k-1} F_{k}}
$$

It is clear that

$$
\sum_{k=n+2}^{m n} \frac{(-1)^{k-1}}{F_{k-2} F_{k-1} F_{k}}>0
$$

Since $m \geq 3$ and invoking (2.8), we obtain

$$
F_{m n-1}\left(F_{n-3}+F_{n}\right) \geq F_{3 n-1}\left(F_{n-3}+F_{n}\right)>F_{n-2} F_{n-1} F_{n} F_{n+1},
$$

which implies

$$
\begin{aligned}
\frac{1}{F_{n-2} F_{n-1} F_{n}}-\frac{1}{F_{n-1} F_{n} F_{n+1}}-\frac{1}{F_{m n-1}} & =\frac{F_{n+1}-F_{n-2}}{F_{n-2} F_{n-1} F_{n} F_{n+1}}-\frac{1}{F_{m n-1}} \\
& =\frac{F_{n-3}+F_{n}}{F_{n-2} F_{n-1} F_{n} F_{n+1}}-\frac{F_{n-3}+F_{n}}{F_{m n-1}\left(F_{n-3}+F_{n}\right)} \\
& >0 .
\end{aligned}
$$

Therefore, (2.13) also holds for $n \geq 5$.

Now we state our main results on the sum of reciprocal Fibonacci numbers.

Theorem 2.10 For all $n \geq 4$, we have

$$
\left\lfloor\left(\sum_{k=n}^{2 n} \frac{1}{F_{k}}\right)^{-1}\right\rfloor=F_{n-2}
$$

Proof Combining (2.9), (2.10), and (2.12), we conclude that, for all $n \geq 4$,

$$
\frac{1}{F_{n-2}+1}<\sum_{k=n}^{2 n} \frac{1}{F_{k}}<\frac{1}{F_{n-2}}
$$

from which (2.14) follows immediately.

Remark Identity (2.14) was first conjectured by Professor Ohtsuka, the first author of [10]. Based on the formula of $F_{n}$ and using analytic methods, Wu and Wang [14] presented a proof of (2.14). In contrast to Wu and Wang's work, the techniques we use here are more elementary. 
Theorem 2.11 If $m \geq 3$ and $n \geq 2$, then

$$
\left\lfloor\left(\sum_{k=n}^{m n} \frac{1}{F_{k}}\right)^{-1}\right\rfloor= \begin{cases}F_{n-2}, & \text { if } n \text { is even; } \\ F_{n-2}-1, & \text { if } n \text { is odd. }\end{cases}
$$

Proof It is clear that

$$
\left\lfloor\left(\sum_{k=2}^{2 m} \frac{1}{F_{k}}\right)^{-1}\right\rfloor=F_{0}
$$

Combining (2.9) and (2.10), we find that, for all even integers $n \geq 4$,

$$
\frac{1}{F_{n-2}+1}<\sum_{k=n}^{m n} \frac{1}{F_{k}}<\frac{1}{F_{n-2}}
$$

Thus (2.16) and (2.17) show that, for all $m \geq 3$,

$$
\left\lfloor\left(\sum_{k=n}^{m n} \frac{1}{F_{k}}\right)^{-1}\right\rfloor=F_{n-2}
$$

provided that $n \geq 2$ is even.

Next we aim to prove that, for $m \geq 3$ and all odd integers $n \geq 3$,

$$
\left\lfloor\left(\sum_{k=n}^{m n} \frac{1}{F_{k}}\right)^{-1}\right\rfloor=F_{n-2}-1
$$

If $n=3$, we can readily see that

$$
\sum_{k=3}^{3 m} \frac{1}{F_{k}}>1
$$

thus (2.18) holds for $n=3$. So in the rest of the proof we assume that $n \geq 5$.

It is not hard to derive that, for all $k \geq 5$,

$$
\frac{1}{F_{k-2}-1}-\frac{1}{F_{k}}-\frac{1}{F_{k-1}-1}=\frac{(-1)^{k}-1+F_{k}}{F_{k}\left(F_{k-2}-1\right)\left(F_{k-1}-1\right)}>0 .
$$

Hence, we get

$$
\sum_{k=n}^{m n} \frac{1}{F_{k}}<\frac{1}{F_{n-2}-1}-\frac{1}{F_{m n-1}-1}<\frac{1}{F_{n-2}-1}
$$

Finally, combining (2.19) with (2.13) yields (2.18).

Remark As $m \rightarrow \infty,(2.15)$ becomes (1.1). Hence our result, Theorem 2.11, substantially improves Theorem 1.1. 


\section{Reciprocal square sum of the Fibonacci numbers}

We first give several preliminary results which will be used in our later proofs.

Lemma 3.1 For all $n \geq 1$,

$$
F_{n} F_{n+1}-F_{n-1} F_{n+2}=(-1)^{n-1} .
$$

Proof It is easy to show that

$$
\begin{aligned}
F_{n} F_{n+1}-F_{n-1} F_{n+2} & =F_{n} F_{n+1}-F_{n-1}\left(F_{n}+F_{n+1}\right) \\
& =F_{n} F_{n+1}-F_{n} F_{n-1}-F_{n-1} F_{n+1} \\
& =F_{n}\left(F_{n+1}-F_{n-1}\right)-F_{n-1} F_{n+1} \\
& =F_{n}^{2}-F_{n-1} F_{n+1} .
\end{aligned}
$$

Employing (2.1), the desired result follows.

Proposition 3.2 Given an integer $m \geq 2$ and let $n \geq 3$ be odd, we have

$$
\sum_{k=n}^{m n} \frac{1}{F_{k}^{2}}<\frac{1}{F_{n-1} F_{n}} .
$$

Proof It is straightforward to check that, for each $k \geq 2$,

$$
\begin{aligned}
& \frac{1}{F_{k-1} F_{k}}-\frac{1}{F_{k}^{2}}-\frac{1}{F_{k+1}^{2}}-\frac{1}{F_{k+1} F_{k+2}} \\
& =\frac{F_{k} F_{k+1}^{2} F_{k+2}-F_{k-1} F_{k+1}^{2} F_{k+2}-F_{k-1} F_{k}^{2} F_{k+2}-F_{k-1} F_{k}^{2} F_{k+1}}{F_{k-1} F_{k}^{2} F_{k+1}^{2} F_{k+2}} \\
& \quad=\frac{F_{k} F_{k+1}\left(F_{k+1} F_{k+2}-F_{k-1} F_{k}\right)-F_{k-1} F_{k+2}\left(F_{k+1}^{2}+F_{k}^{2}\right)}{F_{k-1} F_{k}^{2} F_{k+1}^{2} F_{k+2}} \\
& =\frac{\left(F_{k} F_{k+1}-F_{k-1} F_{k+2}\right) F_{2 k+1}}{F_{k-1} F_{k}^{2} F_{k+1}^{2} F_{k+2}} \\
& =\frac{(-1)^{k-1} F_{2 k+1}}{F_{k-1} F_{k}^{2} F_{k+1}^{2} F_{k+2}}
\end{aligned}
$$

where the last equality follows from (3.1).

Since $n$ is odd, we have

$$
\frac{1}{F_{n-1} F_{n}}-\frac{1}{F_{n}^{2}}-\frac{1}{F_{n+1}^{2}}-\frac{1}{F_{n+1} F_{n+2}}>0
$$

If $m$ is even, then

$$
\sum_{k=n}^{m n} \frac{1}{F_{k}^{2}}<\frac{1}{F_{n-1} F_{n}}-\frac{1}{F_{m n} F_{m n+1}}<\frac{1}{F_{n-1} F_{n}} .
$$


If $m$ is odd, then

$$
\sum_{k=n}^{m n} \frac{1}{F_{k}^{2}}<\frac{1}{F_{n-1} F_{n}}-\frac{1}{F_{m n-1} F_{m n}}+\frac{1}{F_{m n}^{2}}<\frac{1}{F_{n-1} F_{n}} .
$$

Thus, (3.2) always holds.

Proposition 3.3 Let $n$ be odd, then we have

$$
\sum_{k=n}^{2 n} \frac{1}{F_{k}^{2}}>\frac{1}{F_{n-1} F_{n}+1}
$$

Proof Invoking (2.1), we can readily derive that

$$
\frac{1}{F_{k-1} F_{k}+1}-\frac{1}{F_{k}^{2}}-\frac{1}{F_{k} F_{k+1}+1}= \begin{cases}-\frac{2 F_{k-1} F_{k}+1}{F_{k}^{2}\left(F_{k-1} F_{k}+1\right)\left(F_{k} F_{k+1}+1\right)}, & \text { if } k \text { is odd } \\ -\frac{2 F_{k} F_{k+1}+1}{F_{k}^{2}\left(F_{k-1} F_{k}+1\right)\left(F_{k} F_{k+1}+1\right)}, & \text { if } k \text { is even. }\end{cases}
$$

Now we have

$$
\begin{aligned}
\sum_{k=n}^{2 n} \frac{1}{F_{k}^{2}}= & \frac{1}{F_{n-1} F_{n}+1}+\left(\frac{2 F_{n-1} F_{n}+1}{F_{n}^{2}\left(F_{n-1} F_{n}+1\right)\left(F_{n} F_{n+1}+1\right)}\right. \\
& +\frac{2 F_{n+1} F_{n+2}+1}{F_{n+1}^{2}\left(F_{n} F_{n+1}+1\right)\left(F_{n+1} F_{n+2}+1\right)}+\cdots \\
& \left.+\frac{2 F_{2 n} F_{2 n+1}+1}{F_{2 n}^{2}\left(F_{2 n-1} F_{2 n}+1\right)\left(F_{2 n} F_{2 n+1}+1\right)}\right)-\frac{1}{F_{2 n} F_{2 n+1}+1} \\
> & \frac{1}{F_{n-1} F_{n}+1}+\frac{2 F_{n-1} F_{n}+1}{F_{n}^{2}\left(F_{n-1} F_{n}+1\right)\left(F_{n} F_{n+1}+1\right)}-\frac{1}{F_{2 n} F_{2 n+1}+1} .
\end{aligned}
$$

It is obvious that $2 F_{k-1} F_{k} \geq F_{k-1} F_{k+1}$. From (2.1) and the fact that $n$ is odd, we obtain

$$
\frac{2 F_{n-1} F_{n}+1}{F_{n}^{2}} \geq \frac{F_{n-1} F_{n+1}+1}{F_{n}^{2}}=\frac{F_{n}^{2}}{F_{n}^{2}}=1,
$$

which implies that

$$
\sum_{k=n}^{2 n} \frac{1}{F_{k}^{2}}>\frac{1}{F_{n-1} F_{n}+1}+\frac{1}{\left(F_{n-1} F_{n}+1\right)\left(F_{n} F_{n+1}+1\right)}-\frac{1}{F_{2 n} F_{2 n+1}+1} .
$$

By (2.3) and (2.4), we have

$$
\begin{aligned}
F_{2 n} F_{2 n+1}+1 & >\left(F_{n-1} F_{n}+F_{n} F_{n+1}\right)\left(F_{n}^{2}+F_{n+1}^{2}\right) \\
& >\left(F_{n-1} F_{n}+1\right)\left(F_{n} F_{n+1}+1\right),
\end{aligned}
$$

from which we conclude that

$$
\sum_{k=n}^{2 n} \frac{1}{F_{k}^{2}}>\frac{1}{F_{n-1} F_{n}+1} .
$$

The proof is complete. 
Proposition 3.4 Suppose that $m \geq 2$ and $n>0$ is even. Then

$$
\sum_{k=n}^{m n} \frac{1}{F_{k}^{2}}<\frac{1}{F_{n-1} F_{n}-1} .
$$

Proof Applying (2.1), we can rewrite $F_{k}^{4}$ as

$$
F_{k}^{4}=F_{k-1} F_{k}^{2} F_{k+1}+(-1)^{k-1} F_{k}^{2} .
$$

In addition,

$$
\begin{aligned}
\left(F_{k-1} F_{k}-1\right)\left(F_{k} F_{k+1}-1\right) & =F_{k-1} F_{k}^{2} F_{k+1}-F_{k-1} F_{k}-F_{k} F_{k+1}+1 \\
& =F_{k-1} F_{k}^{2} F_{k+1}-2 F_{k-1} F_{k}-F_{k}^{2}+1 .
\end{aligned}
$$

Combining (3.5) and (3.6) yields

$$
\begin{aligned}
\frac{1}{F_{k-1} F_{k}-1}-\frac{1}{F_{k}^{2}}-\frac{1}{F_{k} F_{k+1}-1} & =\frac{F_{k}^{2}}{\left(F_{k-1} F_{k}-1\right)\left(F_{k} F_{k+1}-1\right)}-\frac{1}{F_{k}^{2}} \\
& =\frac{2 F_{k-1} F_{k}-1+F_{k}^{2}+(-1)^{k-1} F_{k}^{2}}{\left(F_{k-1} F_{k}-1\right)\left(F_{k} F_{k+1}-1\right) F_{k}^{2}} \\
& \geq \frac{2 F_{k-1} F_{k}-1}{\left(F_{k-1} F_{k}-1\right)\left(F_{k} F_{k+1}-1\right) F_{k}^{2}} \\
& >0 .
\end{aligned}
$$

Therefore,

$$
\sum_{k=n}^{m n} \frac{1}{F_{k}^{2}}<\frac{1}{F_{n-1} F_{n}-1}-\frac{1}{F_{m n} F_{m n+1}-1}<\frac{1}{F_{n-1} F_{n}-1},
$$

which completes the proof.

Proposition 3.5 If $n>0$ is even, then

$$
\sum_{k=n}^{2 n} \frac{1}{F_{k}^{2}}>\frac{1}{F_{n-1} F_{n}}
$$

Proof Employing (2.1), we can deduce that

$$
\frac{1}{F_{k-1} F_{k}}-\frac{1}{F_{k}^{2}}-\frac{1}{F_{k} F_{k+1}}=\frac{(-1)^{k-1}}{F_{k-1} F_{k}^{2} F_{k+1}} \text {. }
$$

Hence, since $n$ is even, we have

$$
\begin{aligned}
\sum_{k=n}^{2 n} \frac{1}{F_{k}^{2}}= & \frac{1}{F_{n-1} F_{n}}+\sum_{k=n}^{2 n} \frac{(-1)^{k}}{F_{k-1} F_{k}^{2} F_{k+1}}-\frac{1}{F_{2 n} F_{2 n+1}} \\
= & \frac{1}{F_{n-1} F_{n}}+\left(\frac{1}{F_{n-1} F_{n}^{2} F_{n+1}}-\frac{1}{F_{n} F_{n+1}^{2} F_{n+2}}-\frac{1}{F_{2 n} F_{2 n+1}}\right) \\
& +\sum_{k=n+2}^{2 n-1} \frac{(-1)^{k}}{F_{k-1} F_{k}^{2} F_{k+1}}+\frac{1}{F_{2 n-1} F_{2 n}^{2} F_{2 n+1}} .
\end{aligned}
$$


It is easy to see that

$$
\sum_{k=n+2}^{2 n-1} \frac{(-1)^{k-1}}{F_{k-1} F_{k}^{2} F_{k+1}}>0
$$

thus

$$
\sum_{k=n}^{2 n} \frac{1}{F_{k}^{2}}>\frac{1}{F_{n-1} F_{n}}+\left(\frac{1}{F_{n-1} F_{n}^{2} F_{n+1}}-\frac{1}{F_{n} F_{n+1}^{2} F_{n+2}}-\frac{1}{F_{2 n} F_{2 n+1}}\right) .
$$

We claim that

$$
\frac{1}{F_{n-1} F_{n}^{2} F_{n+1}}-\frac{1}{F_{n} F_{n+1}^{2} F_{n+2}}-\frac{1}{F_{2 n} F_{2 n+1}}>0 .
$$

First, by (2.6), we have

$$
\frac{1}{F_{n-1} F_{n}^{2} F_{n+1}}-\frac{1}{F_{n} F_{n+1}^{2} F_{n+2}}-\frac{1}{F_{2 n} F_{2 n+1}}=\frac{F_{2 n+1}}{F_{n-1} F_{n}^{2} F_{n+1}^{2} F_{n+2}}-\frac{F_{2 n+1}}{F_{2 n} F_{2 n+1}^{2}} .
$$

It follows from (2.3), (2.4), and (2.5) that

$$
\begin{aligned}
& F_{2 n}>F_{n-1} F_{n}, \\
& F_{2 n+1}>F_{n+1}^{2}, \\
& F_{2 n+1}>F_{n} F_{n+2},
\end{aligned}
$$

which implies that

$$
F_{2 n} F_{2 n+1}^{2}>F_{n-1} F_{n}^{2} F_{n+1}^{2} F_{n+2} \text {. }
$$

Thus we obtain

$$
\frac{1}{F_{n-1} F_{n}^{2} F_{n+1}}-\frac{1}{F_{n} F_{n+1}^{2} F_{n+2}}-\frac{1}{F_{2 n} F_{2 n+1}}>0,
$$

which yields the desired (3.7).

Now we introduce our main result on the square sum of reciprocal Fibonacci numbers.

Theorem 3.6 For all $n \geq 1$ and $m \geq 2$, we have

$$
\left\lfloor\left(\sum_{k=n}^{m n} \frac{1}{F_{k}^{2}}\right)^{-1}\right\rfloor= \begin{cases}F_{n} F_{n-1}, & \text { if } n \text { is odd } \\ F_{n} F_{n-1}-1, & \text { if } n \text { is even } .\end{cases}
$$

Proof We first consider the case when $n$ is odd. If $n=1$, the result is clearly true. So we assume that $n \geq 3$.

It follows from (3.3) that

$$
\sum_{k=n}^{m n} \frac{1}{F_{k}^{2}} \geq \sum_{k=n}^{2 n} \frac{1}{F_{k}^{2}}>\frac{1}{F_{n-1} F_{n}+1} .
$$


Employing (3.2) and (3.9) yields

$$
\frac{1}{F_{n-1} F_{n}+1}<\sum_{k=n}^{m n} \frac{1}{F_{k}^{2}}<\frac{1}{F_{n-1} F_{n}}
$$

which implies that, if $n>0$ is odd, we have

$$
\left\lfloor\left(\sum_{k=n}^{m n} \frac{1}{F_{k}^{2}}\right)^{-1}\right\rfloor=F_{n} F_{n-1} .
$$

We now consider the case where $n>0$ is even. It follows from (3.7) that

$$
\sum_{k=n}^{m n} \frac{1}{F_{k}^{2}} \geq \sum_{k=n}^{2 n} \frac{1}{F_{k}^{2}}>\frac{1}{F_{n-1} F_{n}} .
$$

Combining (3.4) and (3.10), we arrive at

$$
\frac{1}{F_{n-1} F_{n}}<\sum_{k=n}^{m n} \frac{1}{F_{k}^{2}}<\frac{1}{F_{n-1} F_{n}-1}
$$

from which we find that, if $n>0$ is even,

$$
\left\lfloor\left(\sum_{k=n}^{m n} \frac{1}{F_{k}^{2}}\right)^{-1}\right\rfloor=F_{n} F_{n-1}-1 .
$$

This completes the proof.

Remark Theorem 1.2 can be regarded as the limiting case as $m \rightarrow \infty$ in (3.8).

\section{Competing interests}

The authors declare that they have no competing interests.

\section{Authors' contributions}

All authors contributed equally to deriving all the results of this article, and read and approved the final manuscript.

\section{Acknowledgements}

This work was supported by the National Natural Science Foundation of China (No. 11401080) and the Research Project of Education Teaching Reform of University of Electronic Science and Technology of China (No. 2013XJYEL032). The authors would like to thank the referees for helpful comments leading to an improvement of an earlier version.

Received: 14 November 2014 Accepted: 12 February 2015 Published online: 28 February 2015

\section{References}

1. Sloane, NJA: The On-Line Encyclopedia of Integer Sequences. https://oeis.org (1991). Accessed 30 Apr 1991

2. Duncan, RL: Applications of uniform distribution to the Fibonacci numbers. Fibonacci O. 5, 137-140 (1967)

3. Karaduman, E: An application of Fibonacci numbers in matrices. Appl. Math. Comput. 147, 903-908 (2004)

4. Ma, R, Zhang, WP: Several identities involving the Fibonacci numbers and Lucas numbers. Fibonacci Q. 45, 164-170 (2007)

5. Vorobiev, NN: Fibonacci Numbers. Springer, Basel (2002)

6. Elsner, C, Shimomura, S, Shiokawa, I: Algebraic relations for reciprocal sums of Fibonacci numbers. Acta Arith. 130, 37-60 (2007)

7. Elsner, C, Shimomura, S, Shiokawa, I: Algebraic relations for reciprocal sums of odd terms in Fibonacci numbers. Ramanujan J. 17, 429-446 (2008)

8. Elsner, C, Shimomura, S, Shiokawa, I: Algebraic independence results for reciprocal sums of Fibonacci numbers. Acta Arith. 148, 205-223 (2011) 
9. Elsner, C, Shimomura, S, Shiokawa, I: Algebraic relations for reciprocal sums of even terms in Fibonacci numbers. J. Math. Sci. 180, 650-671 (2012)

10. Ohtsuka, H, Nakamura, S: On the sum of reciprocal Fibonacci numbers. Fibonacci Q. 46/47, 153-159 (2008/2009)

11. Wu, ZG, Zhang, WP: The sums of the reciprocals of Fibonacci polynomials and Lucas polynomials. J. Inequal. Appl. 2012, Article ID 134 (2012)

12. Wu, ZG, Zhang, WP: Several identities involving the Fibonacci polynomials and Lucas polynomials. J. Inequal. Appl. 2013, Article ID 205 (2013)

13. Holliday, SH, Komatsu, T: On the sum of reciprocal generalized Fibonacci numbers. Integers 11A, Article ID 11 (2011)

14. Wu, ZG, Wang, TT: The finite sum of reciprocal of the Fibonacci numbers. J. Inn. Mong. Norm. Univ. Nat. Sci. 40, 126-129(2011)

Submit your manuscript to a SpringerOpen ${ }^{\circ}$ journal and benefit from:

- Convenient online submission

- Rigorous peer review

- Immediate publication on acceptance

- Open access: articles freely available online

- High visibility within the field

Retaining the copyright to your article

Submit your next manuscript at $\boldsymbol{s p r i n g e r o p e n . c o m ~}$ 